\title{
How a discerning cytological examination can aid in the diagnosis of infectious diseases: case reports
}

\author{
D.K. Faria (ii) ${ }^{1}$, J.N. de Almeida Júnior ${ }^{1}{ }^{1}$, C.S. Faria (iD) ${ }^{2}$, B. Durante ${ }^{1}{ }^{1}$, B.F. Falasco (iD) ${ }^{1}$, \\ E. Terreri Neto (id ${ }^{1}$, and L. Antonangelo (iD) ${ }^{1,2}$ \\ ${ }^{1}$ Laboratório de Patologia Clínica, Departamento de Patologia, Hospital das Clinicas, Faculdade de Medicina, \\ Universidade de São Paulo, São Paulo, SP, Brasil \\ ${ }^{2}$ Laboratório de Investigação Médica, Hospital das Clínicas, Faculdade de Medicina, \\ Universidade de São Paulo, São Paulo, SP, Brasil
}

\begin{abstract}
Infections caused by uncommon and resistant pathogens in unusual sites have been increasingly reported in medical literature. We describe four cases of rare cytological findings and clinical impact for patients. In the first case, Aspergillus sp and Pneumocystis jirovecii were observed in the bronchoalveolar lavage of a patient with severe systemic lupus. In the second and third cases, we describe the presence of Trichomonas sp and Strongyloides sp larvae in samples of pleural and peritoneal fluid, respectively. The fourth report is about a patient with a wrist subcutaneous nodule whose synovial aspiration and cytology revealed the presence of brown septate hyphae. The early identification of the infectious agent in the cytological examination was essential for the introduction and/or re-adaptation of therapy in the four cases described. Patients in this report were immunocompromised with severe comorbidities, conditions often associated with unfavorable clinical outcomes.
\end{abstract}

Key words: Cytology; Bronchoalveolar lavage; Pleural effusion; Ascites; Infectious diseases

\section{Introduction}

The cytological examination of cavity fluids, sputum, and bronchoalveolar lavage $(\mathrm{BAL})$ is part of the diagnostic routine of clinical laboratories, especially in tertiary referral hospitals. Although the exam focuses primarily on the analysis of cell predominance and oncotic cytology, the detection of bacteria, fungi, and parasites is part of the examination and the result is described in the medical report, aiding in the etiologic diagnosis of these unusual cases (1).

Infectious diseases, frequent in all areas of healthcare, are responsible for high rates of morbidity and mortality. For the correct therapeutic approach, the identification of the infectious agent by culture or PCR is the main goal in the clinical setting. However, the positivity of conventional Gram stain and culture in cavity fluids is generally less than $60 \%$ (2). Furthermore, with the increasing consumption of antimicrobial medications and the increasing at-risk population, especially of immunocompromised patients, infections caused by rare and resistant pathogens in unusual sites have been described more frequently in the medical literature (3).

Increased cancer incidence, the global spread of AIDS (acquired immunodeficiency syndrome), and the current availability of solid organ transplants have required a more thorough cytological examination of body fluids and BAL by clinical laboratories $(4,5)$. Collection of these biological samples is not invasive and their analyses can provide results as efficient as those obtained from biopsies in a shorter time (within 24 to $48 \mathrm{~h}$ from collection).

In this report, we describe four cases of infrequent infectious agents found in different types of biological samples during routine cytological examination. In all cases, the cytological findings were essential for the etiological diagnosis and for therapeutic adequacy. The patients were immunocompromised with severe comorbidities, conditions frequently associated with unfavorable clinical outcomes.

\section{Description of cases}

Four clinical cases from the Hospital das Clínicas, University of Sao Paulo Medical School, presenting unusual infectious agents in samples of body fluids are described. Samples were analyzed between January 2015 and September 2017 and included BAL (Case 1), pleural fluid (Case 2), peritoneal fluid (Case 3 ), and an 
aspirate from a wrist subcutaneous nodule (Case 4). Samples were submitted to routine cytological, biochemical (when relevant), and microbiological exams at the Clinical Laboratory, which is accredited by the College of American Pathologists (CAP). Clinical history, imaging exams, and other laboratory tests were used for case descriptions. The study was approved by the local Ethical Committee.

BAL was sampled in a siliconized conical tube; cavity fluids and nodule aspirate were collected in tubes coated with the EDTA anticoagulant. All samples were processed within $4 \mathrm{~h}$ from collection. After macroscopic analysis and counting of nucleated cells in a Neubauer chamber, the samples were cytocentrifuged $\left(365 \mathrm{~g}, 25^{\circ} \mathrm{C}, 10 \mathrm{~min}\right)$ for slide preparation. Slides were stained with Leishman's hematological stain for routine cytology, and cytochemical staining was carried out to complement the etiological investigation with periodic acid-Schiff (PAS) reactive component, silver methenamine stain (Grocott), and FontanaMasson stain.

\section{Case 1}

A 22-year-old female with severe systemic lupus erythematous was hospitalized due to refractory thrombocytopenia and increased proteinuria. On admission, her main complaints were dyspnea and pain on the right hemithorax for the last fifteen days. Chest computed tomography revealed cavitated nodules on the right upper lobes (Figure 1A and B). The nodules were considered non-specific and of probable inflammatory/infectious etiology. The cytological analysis of the BAL showed a predominance of macrophages, a moderate number of lymphocytes and neutrophils, presence of septate hyphae, and yeasts suggestive of Aspergillus sp and Pneumocystis jirovecii (Figure 1C, D, and E). BAL culture identified growth of Aspergillus sp. Laboratory test for tuberculosis was negative and serology for human immunodeficiency virus (HIV) and for hepatitis B and C were non-reactive.

Clinical follow-up. The patient was initially submitted to pulse-therapy with methylprednisolone, followed by immunoglobulin infusion, mycophenolate, hydroxychloroquine, danazol, amphotericin $B$, and sodium piperacillintazobactam. After the identification of $P$. jirovecii in BAL, sulfamethoxazole-trimethoprim was introduced. However, her respiratory functions worsened and evolved to metabolic acidosis. She was referred to the intensive care unit with orotracheal intubation and mechanical ventilation. Despite the therapeutic approaches, the patient died in six days.

\section{Case 2}

A 55-year-old female was hospitalized due to a consumptive syndrome without defined etiology. She presented a history of dysphagia, weight loss (10 kg), and asthenia for 3 months, evolving with ascites and lower limb edema in the last month. The patient reported being an exsmoker and ex-alcoholic, with no other previous diseases. On physical examination, the patient was in regular clinical condition, pale, anicteric, dehydrated, and emaciated (37.5 $\mathrm{kg}$ ). Abdominal examination revealed bulging flanks suggestive of ascites. She had lower limb edema and oral moniliasis. The upper gastrointestinal endoscopy and biopsy showed moderate gastritis and severe bulboduodenitis, positive for Helicobacter pylori. Analysis of the ascitic fluid obtained by paracentesis revealed the presence of larvae suggestive of Strongyloides in a predominantly neutrophilic

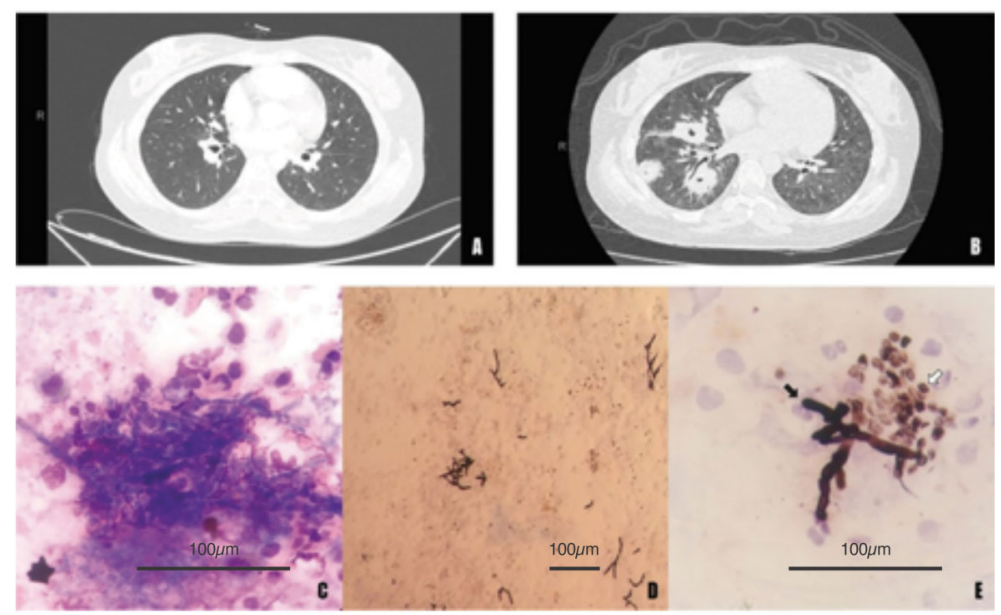

Figure 1. Aspects of chest tomography at admission (A) and after fifteen days of evolution (B) with the presence of excavated nodules with halo signal, initially considered to be of inflammatory/infectious etiology. C, Sample of bronchoalveolar lavage showing presence of macrophages, lymphocytes, neutrophils, and many hyphae and yeasts (Leishman staining, 600×). D and E, Presence of non-specific septate hyphae (black arrow) and yeasts (white arrow) suggestive of Pneumocystis jirovecii (silver methenamine staining, 200 and $600 \times$, respectively). Scale bars: $100 \mu \mathrm{m}$. 

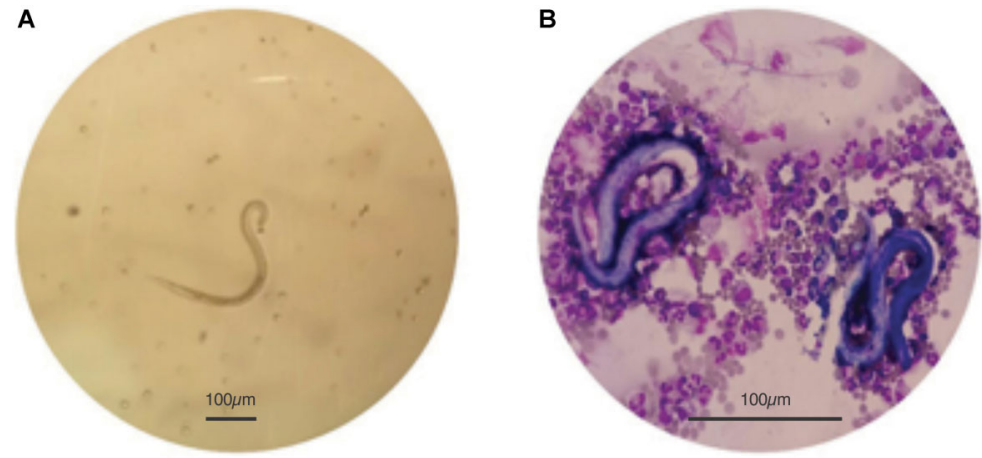

Figure 2. A, Presence of larvae in the fresh analysis of ascitic fluid $(200 \times)$ and B, presence of Strongyloides larvae in a rich neutrophilic fluid (Leishman staining, $600 \times$ ). Scale bars: $100 \mu \mathrm{m}$.

fluid (Figure 2). Parasitological examination of feces (methods of Faust and Lutz/Hoffman) confirmed eggs of Strongyloides stercoralis and in the modified Rugai method, the presence of $S$. stercoralis larvae. The duodenal biopsy showed intense inflammatory reaction and the presence of Strongyloides larvae.

Clinical follow-up. Patient progressed to renal failure, altered mental status, septic shock, and died a few days after admission.

\section{Case 3}

A 46-year-old male, ex-smoker and ex-alcoholic, with a history of squamous cell carcinoma at the esophagogastric transition with liver, bones, and lymph nodes metastases, was hospitalized with complaints of dyspnea, secretory cough, and chest pain. The upper gastrointestinal endoscopy showed a stenotic infiltrative lesion at the esophagogastric transition. Thoracic/abdominal tomography and positron emission tomography (PET) revealed several mediastinal lymph node enlargements (up to $4.2 \mathrm{~cm}$ ) in the transverse axis and parietal thickening of the esophagogastric transition, the liver with small hypodense nodules in segments II and V, a solid and heterogeneous retroperitoneal mass medially to the small gastric curvature $(6.7 \times 4.7 \mathrm{~cm})$, and right pleural effusion. The patient was diagnosed with pneumonia, complicated pleural effusion, and superior vena cava compression syndrome. Antibiotic therapy with piperacillin-tazobactam and vancomycin and full anticoagulation were introduced. Thoracentesis was performed and analysis of the pleural fluid showed flagellate elements suggestive of Trichomonas sp (Figure 3).

Clinical follow-up. Despite metronidazole therapy, his clinical condition worsened and he died. Unfortunately, there was a limitation for the identification of the species of Trichomonas sp.

\section{Case 4}

A 60-year-old male rural worker, ex-smoker, with a previous history of hypothyroidism, epilepsy, chronic renal

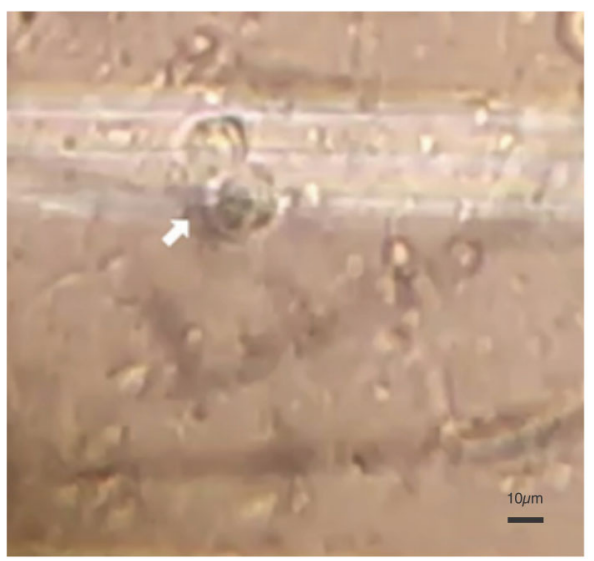

Figure 3. Pleural fluid fresh examination $(600 \times$, scale bar: $10 \mu \mathrm{m})$ showing flagellated forms of Trichomonas sp.

failure, and cardiac transplantation due to dilated Chagas cardiomyopathy, sought medical assistance due to an increasing cyst on the left wrist. The patient was using immunosuppressive therapy (mycophenolate sodium, cyclosporin, and prednisone). On examination, the cyst was mobile, well-delimited, painful, and without phlogistic signs. Cytological analysis of the cystic fluid revealed moderate cellularity with a predominance neutrophils and macrophages and the presence of many brown septate hyphae. Fontana-Masson staining showed melanin-positive hyphae (Figure 4A, B, and C); the mycological culture confirmed the growth of melanized fungal colonies, with hyphae and spore in Sabouraud agar medium (Figure 4D). The ultrasoundguided biopsy revealed a chronic inflammatory process with foci of suppurative acute activity and the presence of hyphae and fungal spores, confirming the cytological findings.

Clinical follow-up. After the laboratory results, itraconazole was introduced to the patient and he is currently controlled and in outpatient follow-up. 

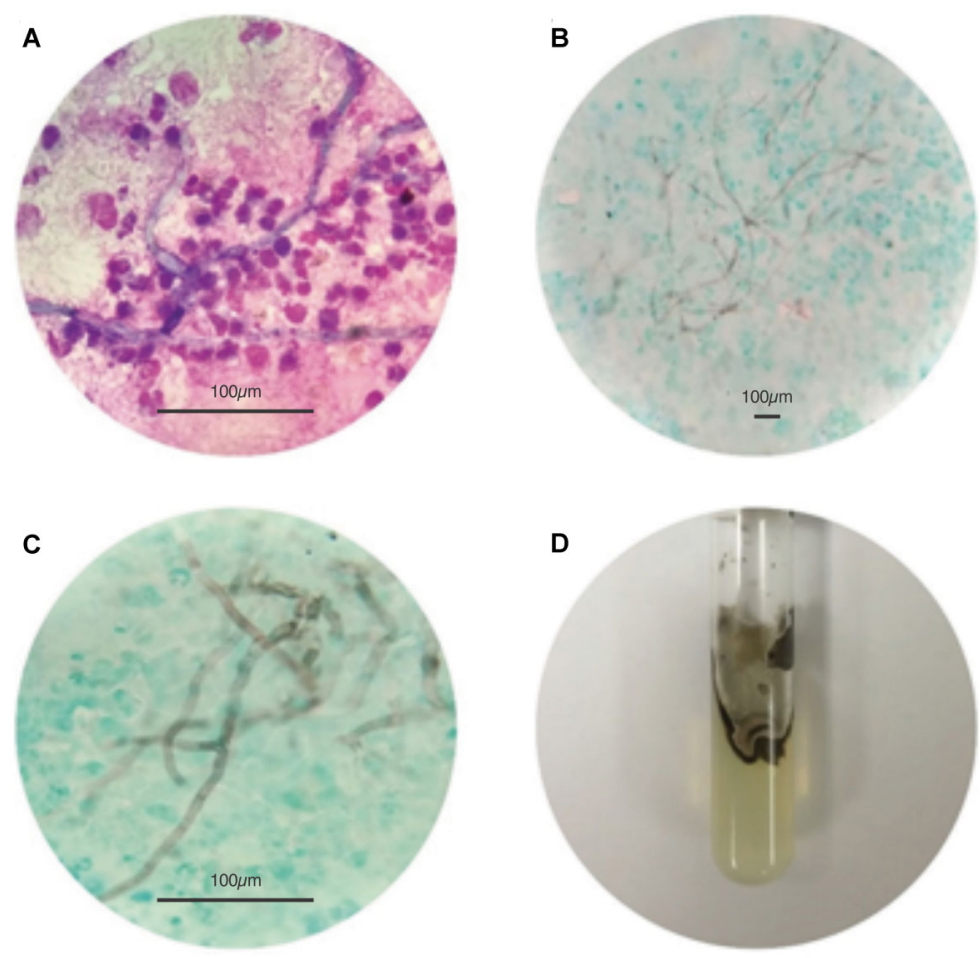

Figure 4. Presence of brown septate hyphae in the cytological examination of the cystic content with Leishman staining $(600 \times)(\mathbf{A})$ and Fontana-Masson staining, $100 \times(B)$ and $600 \times(C)$, respectively. Scale bars: $100 \mu \mathrm{m}$. D. Aspect of fungal growth in Sabouraud agar medium.

\section{Discussion}

\section{Co-infection of Aspergillus sp and Pneumocystis jirovecii in BAL sample}

Aspergillus sp, mainly Aspergillus fumigatus, is a ubiquitous saprophytic fungus transmitted by inhalation of spores or conidia. The mycosis can be manifested in several clinical forms: invasive, chronic necrotizing, allergic bronchopulmonary, and aspergilloma (6). Invasive aspergillosis is a frequent cause of death in immunocompromised patients, especially in those with hematological disorders (7). Currently, the diagnosis based on biomarkers (galactomannan, polymerase chain reaction, and $\beta$-D-glucan) has significantly improved cytological diagnosis $(8-10)$.

$P$. jirovecii is a yeast fungus that colonizes the lungs, triggering pneumonia in immunocompromised patients, especially in those HIV-infected with T-helper (CD4 +) cell count lesser than 200 cells $/ \mathrm{mm}^{3}$ (11).

The co-infection of $A$. fumigatus and $P$. jirovecii in HIVnegative patients is a rare event. $A$ recently published systematic review identified only 7 cases in the medical literature (12). To date, this is the first case of Aspergillus and $P$. jirovecii co-infection identified in a bronchoalveolar lavage sample in Brazil.

\section{Identification of Strongyloides sp in ascites}

Strongyloidiasis is a parasitic infection caused by $S$. stercoralis or S. fuelleborni, a nematode widely distributed in the world, especially in tropical areas (13). The diagnosis is usually based on the direct visualization of larvae in feces, with low sensitivity. Enzymelinked immunosorbent assay, western blot, and molecular techniques can be used as complementary methods (14). In adults, the main risk factors associated to strongyloidiasis are related to impairment of the immune system, caused by alcoholism, use of corticoids, neoplasms, HIV infection, and human T-lymphotropic virus 1 $(13,15)$.

The life cycle of $S$. stercoralis alternates between generations of free-living and parasite forms and the host becomes infected through the skin by the filarial larva, initiating an intestinal and pulmonary cycle. In the parthenogenic stage, the nematode deposits eggs in the intestinal mucosa, which hatch and release rhabditoid larvae that develop or mate in the soil or directly self-infect the host through the terminal intestinal mucosa $(13,16)$. Infected patients can be asymptomatic or present cutaneous, gastrointestinal (diarrhea, abdominal discomfort, nausea, anorexia), and/or pulmonary symptoms, or can even present more severe symptoms of hyperinfection 
Table 1. Literature data concerning the finding of Strongyloides stercoralis in peritoneal fluid.

\begin{tabular}{|c|c|c|c|c|}
\hline Case report & Country & Gender (age in years) & Underlying condition & Clinical outcome \\
\hline Avagnina et al., 1980 (18) & Argentina & Male (33) & Renal transplantation & Death \\
\hline Hong et al., 2004 (19) & United States & Male (49) & HIV-positive & Favorable \\
\hline Sav et al., 2009 (20) & Turkey & Male (67) & Peritoneal dialysis & Favorable \\
\hline Saha et al., 2012 (21) & United States & Male (59) & Peritoneal dialysis & Favorable \\
\hline Shukla et al., 2015 (22) & India & Male (24) & Diabetes and alcoholic & - \\
\hline Zhang et al., 2017 (23) & China & Female (57) & Peritoneal dialysis & Death \\
\hline
\end{tabular}

HIV: human immunodeficiency virus.

(17). The finding of $S$. stercoralis in peritoneal fluid is a rare event, with only 6 cases reported in a recent literature review (Table 1).

\section{Trichomonas sp in pleural fluid}

Trichomonas $\mathrm{sp}$ is a group of flagellate protozoa, with four species infecting humans: Trichomonas vaginalis, Dientamoeba fragilis, Pentatrichomonas hominis, and Trichomonas tenax (24). The infection caused by Trichomonas vaginalis is a common sexually transmitted disease, with 280 million new cases worldwide. Patients are usually asymptomatic (50-70\%), although women may present vaginal pruritus and discharge, vulvovaginitis with erosive lesions, and edema with hemorrhagic areas on the cervical wall, increasing the risk for concomitant HIV infection $(25,26)$. P. hominis (formerly called Trichomonas hominis or Trichomonas intestinalis) lives by commensalism in the intestine, usually causing asymptomatic infection; however, it can cause diarrhea in immunocompromised patients (24). $D$. fragilis has been associated with recurrent abdominal pain in children, irritable bowel syndrome in adults, and increased risk of intestinal infections in patients using antibiotics (27). T. tenax is associated with oral cavity infections. In a systematic review of 47 studies, the presence of gingivitis and periodontitis ranged from 0 to $94.1 \%(28)$.

Pulmonary infection by Trichomonas $\mathrm{sp}$ is an uncommon event, more associated with T. tenax (29). According to Leterrier et al. (30), only 17 cases of Trichomonas sp were identified in pleural fluid according to the medical literature since 1965. In Brazil, we did not find any previous report.

\section{Phaeohyphomycosis in wrist nodule}

Phaeohyphomycosis is an opportunistic fungal infection caused by filamentous species that have brown melanin pigments in their cell walls $(31,32)$. It is an uncommon cause of disease in humans, although it can cause infections in immunocompromised individuals (32). Epidemiological data are scarce in the medical literature. Rees et al. (33) reported the incidence of $1: 1,000,000$ cases per year in San Francisco, in the United States.
Cutaneous or subcutaneous nodules are the most common form of the infection, which presents an indolent course (34). The infection results from the inoculation of the fungal agent through the skin by minor trauma when in contact with contaminated soil, plants, or wood $(33,34)$. Revankar et al. (35) reported episodes of fever (76\%), pulmonary infections (46\%), cutaneous manifestations $(33 \%)$, such as rashes and ulcers, cardiac infections $(29 \%)$, central nervous system infections $(22 \%)$, and urinary infections $(22 \%)$ in 72 patients studied. Some cases of scrotal pouch infection have been reported (3638). In our report, the identification of the black fungus excluded the suspicion of wrist neoplasia and provided the basis for the adequate surgical treatment with patient clinical improvement.

Regarding the complexity of the reported cases, the results of the cytological examination were released in less than $48 \mathrm{~h}$, and patient management was complemented/reconsidered after medical contact. Most clinical laboratories report only the leukocyte differential in the cytological examination of cavity fluids/BAL, referring microbiological, molecular, and oncotic cytology to Microbiology, Molecular Biology, and Pathological Anatomy laboratories, delaying the release of exam results.

The results of fungal or mycobacterial culture take days or months, and not all laboratories have a wide molecular menu in their routines; thus, we reinforce that the cytological examination should be quantitative (total nucleated cells and respective percentages), with research of anomalous elements, such as fungi, bacteria, tumor cells, etc. The double centrifugation used in the preparation of the slides favors the finding of rarer elements, allowing the presumptive diagnosis generally in $24-48 \mathrm{~h}$. The immediate communication of the cytological findings can result in therapeutic adequacy, which is often fundamental to the patient's clinical outcome.

\section{Final remarks}

In the present study, we described a series of cases whose etiological diagnoses were triggered by routine 
cytological findings. In all cases, identification of the infectious agent was essential for the introduction and/ or re-adaptation of therapy. The cases were related to immunocompromised patients or patients with severe comorbidities, conditions often associated with unfavorable clinical outcomes.

\section{References}

1. Michel CW, Davidson B. Pre-analytical issues in effusion cytology. Pleura Peritoneum 2016; 1: 45-56, doi: 10.1515/ pp-2016-0001.

2. Menzies SM, Rahman NM, Wrightson JM, Davies HE, Shorten R, Gillespie SH, et al. Blood culture bottle culture of pleural fluid in pleural infection. Thorax 2011; 66: 658-662, doi: $10.1136 /$ thx.2010.157842.

3. Zuccotti G, Fontanarosa PB. Progress in infectious disease and immunology. JAMA 2011; 305: 1486-1487, doi: 10.1001/ jama.2011.452.

4. Xing LY, Yin J, Shao M, Yang YL, Li KY, Xue MM, et al. Clinical characteristics and prognosis of serous body cavity effusions in patients with sepsis: a retrospective observational study. BMC Anesthesiol 2018; 18: 169, doi: 10.1186/ s12871-018-0621-6.

5. Antonangelo L, Vargas FS, Acencio MMP, Corá AP, Teixeira $L R$, Genofre EH, et al. Effect of temperature and storage time on cellular analysis of fresh pleural fluid samples. Cytopathology 2012; 23: 103-107, doi: 10.1111/j.13652303.2011.00863.x.

6. Zmeili OS, Soubani AO. Pulmonary aspergillosis: a clinical update. QJM 2007; 100: 317-334, doi: 10.1093/qjmed/ hcm035.

7. Morrissey CO, Chen SCA, Sorrell TC, Milliken S, Bardy PG, Bradstock KF, et al. Galactomannan and PCR versus culture and histology for directing use of antifungal treatment for invasive aspergillosis in high-risk haematology patients: a randomised controlled trial. Lancet Infect Dis 2013; 13: 519-528, doi: 10.1016/S1473-3099(13)70076-8.

8. Zhang S, Wang S, Wan Z, Li R, Yu. The diagnosis of invasive and noninvasive pulmonary aspergillosis by serum and bronchoalveolar lavage fluid galactomannan assay. Biomed Res Int 2015; 2015: 943691, doi: 10.1155/2015/ 943691.

9. Kosmidis C, Denning DW. The clinical spectrum of pulmonary aspergillosis. Thorax 2015; 70: 270-277, doi: 10.1136/thoraxjnl-2014-206291.

10. De Pauw B, Walsh TJ, Donnelly JP, Stevens DA, Edwards $\mathrm{JE}$, Calandra T, et al. Revised definitions of invasive fungal disease from the European Organization for Research and Treatment of Cancer/Invasive Fungal Infections Cooperative Group and the National Institute of Allergy and Infectious Diseases Mycoses Study Group (EORTC/MSG) Consensus Group. Clin Infect Dis 2008; 46: 1813-1821, doi: 10.1086/ 588660.

11. Thomas Jr CF, Limper AH. Pneumocystis pneumonia. N Engl J Med 2004; 350: 2487-2498.

12. Markantonatou AM, loakimidou A, Arvaniti K, Manou E, Papadopoulos V, Kiriklidou T, et al. Pulmonary co-infections by Pneumocystis jirovecii and Aspergillus fumigatus in

\section{Acknowledgments}

We thank the staff team of the Cytology and Microbiology Laboratories from the Clinical Pathology Division, Hospital das Clínicas of the Faculdade de Medicina of Universidade de São Paulo.

non-HIV patients: a report of two cases and literature review. Mycoses 2017; 60: 626-633, doi: 10.1111/myc. 12642.

13. Schär F, Trostdorf U, Giardina F, Khieu V, Muth S, Marti H, et al. Strongyloides stercoralis: global distribution and risk factors. PLoS Negl Trop Dis 2013; 7: e2288, doi: 10.1371/ journal.pntd.0002288.

14. Corral MA, Paula FM, Meisel DMCL, Castilho VLP, Gonçalves EMN, Levy D, et al. Potential immunological markers for diagnosis of human strongyloidiasis using heterologous antigens. Parasitology 2017; 144: 124-130, doi: 10.1017/S0031182016001645.

15. Amor A, Rodriguez E, Saugar JM, Arroyo A, LópezQuintana B, Abera B, et al. High prevalence of Strongyloides stercoralis in school-aged children in a rural highland of north-western Ethiopia: the role of intensive diagnostic workup. Parasit Vectors 2016; 9: 617, doi: 10.1186/s13071-0161912-8.

16. Hunt VL, Tsai IJ, Coghlan A, Reid AJ, Holroyd N, Foth BJ, et al. The genomic basis of parasitism in the Strongyloides clade of nematodes. Nat Genet 2016; 48: 299-307, doi: 10.1038/ng.3495.

17. Ericsson CD, Steffen R, Siddiqui AA, Berk SL. Diagnosis of Strongyloides stercoralis infection. Clin Infect Dis 2001; 7: 1040-1047, doi: 10.1086/322707.

18. Avagnina MA, Elsner B, lotti RM, Re E. Strongyloides stercoralis in Papanicolaou-stained smears of ascitic fluid. Acta Cytol 1980; 24: 36-39.

19. Hong IS, Zaidi SY, McEvoy P, Neafie RC. Diagnosis of Strongyloides stercoralis in a peritoneal effusion from an HIV-seropositive man. A case report. Acta Cytol 2004; 48: 211-214, doi: 10.1159/000326318.

20. Sav T, Yaman O, Gunal Al, Oymak O, Utas C. Peritonitis associated with Strongyloides stercoralis in a patient undergoing continuous ambulatory peritoneal dialysis. NDT Plus 2009; 5: 390-391, doi: 10.1093/ndtplus/sfp084.

21. Saha S, Sengsayadeth S, Golper TA. Intestinal Strongyloides causing peritoneal eosinophilia in peritoneal dialysis. Clin Kidney J 2012; 5: 579-581, doi: 10.1093/ckj/sfs134.

22. Shukla S, Chauhan R, Wadhwa S, Sehgal S, Singh S. Strongyloides stercoralis hyperinfection causing eosinophilic ascites. Diagn Cytopathol 2015; 43: 731-733, doi: 10.1002/ dc. 23281 .

23. Zhang H, Ye H, Pan B, Shao S, Chen D. Strongyloides stercoralis infection in the peritoneal cavity of a patient receiving PD. Perit Dial Int 2017; 37: 662, doi: 10.3747 I pdi.2017.00107.

24. Compaoré C, Kemta Lekpa F, Nebie L, Naimba P, Niakara A. Pentatrichomonas hominis infection in rheumatoid arthritis treated with adalimumab. Rheumatology 2013; 52: 15341535, doi: 10.1093/rheumatology/kes385. 
25. Fonseca THS, Oliveira FMS, Alacoque M, Rocha MI, Leite HV, Santos JFG, et al. (2017). Immunocytochemistry Improving the diagnosis of Trichomonas vaginalis infections. Biomed Res Int.5642535, doi: 10.1155/2017/5642535.

26. Van der Pol B. Clinical and laboratory testing for Trichomonas vaginalis infection. J Clin Microbiol 2016; 54: 7-12, doi: 10.1128/JCM.02025-15.

27. Röser D, Simonsen J, Nielsen HV, Stensvold CR, Mølbak K. History of antimicrobial use and the risk of Dientamoeba fragilis infection. Eur J Clin Microbiol Infect Dis 2015; 34: 1145-1151, doi: 10.1007/s10096-015-2334-9.

28. Marty $M$, Lemaitre $M$, Kémoun $P$, Morrier $J J$, Monsarrat $P$. Trichomonas tenax and periodontal diseases: a concise review. Parasitology 2017; 144: 1417-1425, doi: 10.1017/ S0031182017000701.

29. Memik F. Trichomonads in pleural effusion. JAMA 1968; 204: 1145-1146, doi: 10.1001/jama.1968.03140250125023.

30. Leterrier M, Morio F, Renard BT, Poirier AS, Meigeville M, Chambreuil G. Trichomonads in pleural effusion: case report, literature review and utility of PCR for species identification. New Microbiol 2012; 35: 83-87.

31. Al-Abdely HM. Management of rare fungal infections. Curr Opin Infect Dis 2004; 17: 527-532, doi: 10.1097/00001432200412000-00004.

32. Silveira F, Nucci M. Emergence of black molds in fungal disease: epidemiology and therapy. Curr Opin Infect Dis 2001; 14: 679-684, doi: 10.1097/00001432-20011200000003.
33. Rees JR, Pinner RW, Hajjeh RA, Brandt ME, Reingold AL. The epidemiological features of invasive mycotic infections in the San Francisco Bay area, 1992-1993: results of population-based laboratory active surveillance. Clin Infect Dis 1998; 27: 1138-1147, doi: 10.1093/clinids/27.5.1138.

34. Revankar SG, Patterson JE, Sutton DA, Pullen R, Rinaldi MG. Disseminated phaeohyphomycosis: review of an emerging mycosis. Clin Infect Dis 2002; 34: 467-476, doi: $10.1086 / 338636$.

35. Revankar SG, Kirkpatrick WR, McAtee RK, Fothergill AW, Redding SW, Rinaldi MG, et al. Interpretation of trailing endpoints in antifungal susceptibility testing by the National Committee for Clinical Laboratory Standards method. J Clin Microbiol 1998; 36: 153-156, doi: 10.1128/JCM.36.1.153156.1998.

36. Duvic M, Lowe L, Rios A, MacDonald E, Vance P. Superficial phaeohyphomycosis of the scrotum in a patient with acquired immunodeficiency syndrome. Arch Dermatol 1987; 123: 1597-1599, doi: 10.1001/archderm.1987.01660360025004.

37. Flynn BJ, Bourbeau PP, Cera PJ, Scicchitano LM, Jordan RL, Yap WT. Phaeohyphomycosis of the Epididymis caused by Exophiala jeanselmei. J Urol 1999; 162: 492-493, doi: 10.1016/S0022-5347(05)68602-8.

38. Rosseto AL, Dellatorre G, Pérsio RA, Romeiro JCM, Cruz RCB. Subcutaneous phaeohyphomycosis on the scrotum caused by Exophiala jeanselmei: case report. An Bras Dermatol 2010; 85: 517-520, doi: 10.1590/S0365-05962 010000400013 